\title{
Deutschland: \\ Neues Psychotherapiegesetz vor Abschluss
}

\section{Peter Schulthess}

In Deutschland ist eine Revision des Psychotherapiegesetzes (PsyG) im Gange, die europaweit wohl einen grossen Impact haben wird. Die bisherige Regelung, die erst einen Studienabschluss in Psychologie verlangte, um anschliessend eine Weiterbildung in Psychotherapie zu absolvieren, soll durch ein Gesetz abgelöst werden, das ein Direktstudium in Psychotherapie vorsieht. Es handelt sich um ein fünfjähriges wissenschaftliches Masterstudium, das zur Approbation (Erlaubnis zur Behandlung) führt, und so den Berufseintritt ermöglicht. Es ist gegliedert in eine dreijährige Bachelor- und eine zweijährige Masterstufe.

Inhaltliche Überschneidungen zwischen Psychologiestudium und Weiterbildung entfallen, was Platz schafft für den Einbezug weiterer psychotherapierelevanter Grundlagenfächer aus anderen Sozial- und Kulturwissenschaften, etwa in dem Spektrum, wie es das Ergänzungsstudium Psychotherapiewissenschaft der Charta bzw. der Universitätslehrgang Psychotherapeutische Psychologie der Donau Universität Krems in Zusammenarbeit mit der Charta auch vermittelte. Das Studium der Psychotherapie wird ausschliesslich an Universitäten und ihnen gleichgestellten Hochschulen vermittelt.

Es vereint die Vermittlung von Grundlagenwissen und Psychotherapieausbildung. Im Studium werden alle wissenschaftlich fundierten, evidenzbasierten Therapierichtungen gelehrt werden, auch solche, die nahe dabei sind, als wissenschaftlich fundiert zu gelten. Der Berufstitel wird neu PsychotherapeutIn lauten und löst die Titel Psychologische/r PsychotherapeutIn bzw. Kinder- und Jugendlichenpsychotherapeut/In ab.

Auf der Grundlage der Approbation kann die verfahrensorientierte und altersgruppenspezifische Weiterbildung begonnen werden. An das Studium schliesst ausserdem eine nach jeweiligem Landesrecht organisierte Weiterbildung in stationären oder ambulanten Einrichtungen an. Behandlungsleistungen von PsychotherapeutInnen in Weiterbildung (PiW) werden von den Krankenkassen vergütet. Mit Abschluss der Weiterbildung sind PsychotherapeutInnen berechtigt, sich ins Arztregister eintragen zu lassen und sich um eine Zulassung für die Versorgung im System der gesetzlichen Krankenkassen zu bewerben.
Das Bundesministerium für Gesundheit legte am 3. Januar 2019 einen entsprechenden Referentenentwurf vor, der am 27. Februar 2019 vom Bundeskabinett verabschiedet wurde. Es braucht noch der Genehmigung durch den Bundesrat. Das Gesetz soll in der zweiten Jahreshälfte 2019 verkündet werden. Die ersten Direktstudiengänge sollen im Wintersemsemester 2020/21 starten. Weitere Informationen sind auffindbar unter: https://www.bundesgesundheitsministerium.de /psychotherapeutenausbildung.html

\section{Kommentar}

Europaweit setzt sich eine Akademisierung der Psychotherapie-Ausbildung durch. Vorreiterin eines Direktstudiums in Psychotherapie ist unter anderen die Sigmund Freud Privat Universität Wien, die in Österreich und anderen europäischen Ländern die Studienrichtung Psychotherapiewissenschaft lehrt. In Deutschland folgen nun auch andere Universitäten und Psychologische Fakultäten diesem Modell.

Die Schweiz wird wohl wie üblich mit ein paar Jahren Verzögerung nach Deutschland und anderen europäischen Ländern ihre gesetzliche Regelung entsprechend anpassen müssen. In Deutschland wurde seinerzeit weitsichtig ein Gesetz zur Regelung der Psychologie erlassen und ein anderes zur Regelung der Psychotherapie. Die ASP forderte anlässlich der Gesetzgebungsarbeiten ebenfalls ein separates Psychologiegesetz und ein Psychotherapiegesetz, um den Beruf des/r PsychotherapeutIn als selbstständigen Beruf zu regeln. Die Schweiz wollte dies jedoch nicht und zog es vor, in nur einem Gesetz die Psychotherapie als Psychologieberuf zu definieren, so wie früher die Medizin die Psychotherapie als ärztlichen Beruf definierte.

Es war für die Behörden und für die Politik zu verführerisch, vereinfachend eine logische Verbindung von Psychologie mit Psychotherapie herzustellen, analog zur Verbindung Medizinstudium-PsychiaterIn/PsychotherapeutIn, um die Diskussion der Psychotherapie als eigenständigem wissenschaftlichen Beruf mit Zugangsmöglichkeiten auch aus anderen sozialen Studienabschlüssen zu beenden. Unberücksichtigt blieb

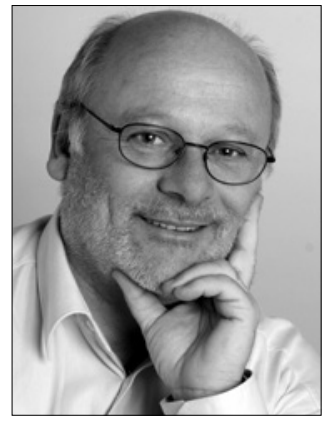


dabei, dass das medizinische Staatsexamen eine Berufszulassung zur ärztlichen Tätigkeit bedeutet, das Medizinstudium also eine Berufsausbildung in einem Heilberuf ist, worauf eine Spezialisierung in einem Teilgebiet der Medizin erfolgt, die zum Facharzttitel führt. Das Studium der Psychologie, auch der klinischen Psychologie, ist anders geartet: Es stellt nicht eine Berufsausbildung in einem Heilberuf dar, der mit dem Studienabschluss ausgeübt werden darf. Die eigentliche Berufsausbildung zur PsychotherapeutIn erfolgt erst in der Weiterbildung. Insofern war die Argumentation der Psychologie-VertreterInnen mit dieser Analogie stets schief, verfing aber. Das neue deutsche Modell löst nun die Psychotherapie von der Pflicht eines Psychologiestudiums ab und regelt sie als eigenständigen Beruf, der in einem Direktstudium erlernt werden kann. Setzt sich das Ausbildungsmodell eines direkten Psychotherapiestudiums, das zugleich eine Berufsausbildung in Psychotherapie darstellt, auch in anderen Ländern durch, so wird die Psychotherapie doch noch ein eigenständiger wissenschaftlicher Beruf, der ab Matura erlernt werden kann und nicht zuerst ein Psychologiestudium voraussetzt.

Die Regelung der Psychotherapie in der Schweiz muss dann wohl aus dem PsyG herausgenommen werden und doch in einem separaten Gesetz geregelt werden. Das hätte man einfacher haben können. ASP und Charta waren wohl der Zeit voraus.

Die Ausbildungszeit und damit die Ausbildungskosten werden mit diesem Modell deutlich verkürzt. Mit dem Masterabschluss kann die Approbation beantragt werden (analog dem Staatsexamen in der Medizin), sieht der deutsche Revisionsentwurf vor, und wer besteht, darf den Beruf der PsychotherapeutIn ab dann ausüben. Eine Spezialisierung auf eine bestimmte Therapierichtung (Vertiefung) oder auf eine bestimmte Altersgruppe oder eine bestimmte Störungsgruppe erfolgt danach und führt zum Titel einer Fachpsychotherapeutin oder eines Fachpsychotherapeuten.

Private Weiterbildungsinstitute werden sich angesichts der Akademisierung der Psychotherapieausbildung Kooperationen mit Uni- versitäten suchen müssen, wollen sie in der Psychotherapieausbildung weiterhin involviert bleiben. Oder sie konzentrieren sich auf postgraduale Spezialisierungen der PsychotherapeutInnen in bestimmten Verfahren oder bezüglich bestimmter Themen. Ausserdem wird auch hier überdeutlich, wie wichtig Forschung ist, die die Evidenzbasierung der gelehrten Therapierichtungen belegen kann.

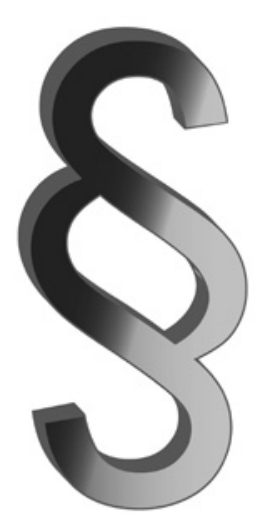

Ich bin zuversichtlich, dass alle Verbände und die Psychologischen Institute diese Entwicklung mittragen werden und solche Direktstudiengänge zur Psychotherapie auch an den Schweizer Hochschulen angeboten werden, sobald sich eine entsprechende Gesetzesänderung abzeichnet. Aber auch da bestehen natürlich politische Unabwägbarkeiten.

Europaweit ist zu ersehen, dass die Akademisierung der Psychotherapieausbildung voranschreitet und auch in weiteren Ländern der Psychotherapieberuf als selbstständiger Beruf geregelt werden wird und Direktstudiengänge das derzeitige Ausbildungsmodell ablösen. Eine europäische Harmonisierung der Psychotherapieregelungen ist nur eine Frage der Zeit.

Peter Schulthess ist Vorstandmitglied der ASP und vertritt diese gemeinsam mit Gabriela Rüttimann in der EAP. In dieser Rolle verfolgt er jeweils die internationale Entwicklung der Psychotherapie. 